\title{
Massive Multi-Agent Simulation in 3D
}

\author{
Masaru Aoyagi and Akira Namatame \\ \{g43037|nama\}@nda.ac.jp \\ National Defense Academy, Japan
}

\begin{abstract}
:
In this paper, we discuss our challenge on how to give the creatures and ability to follow spatial restriction while keeping the complexity low enough to still allow for real-time simulation of the herd. Our methodologies extend the pioneering work by Reynolds' flocking algorithm. We extend how the herd can move in natural-looking paths. Also, we show like the creatures to travel smoothly in 3D space with speed regulation in curve.
\end{abstract}

Keywords: Flocking Behavior, agent, Individual Based Simulation

\section{Introduction}

To creatures a large number of objects by hand would be a tedious job. To make the job "easier, we would like to try to automate as much of the process as possible. For the case of flocks or herds of creatures, Craig Reynolds[1,2] introduced a simple agent-based approach to animate a flock of creatures through space. In this method, each creature makes its own decisions on how to move, according to a small number of simple rules that consider the neighboring members of the flock. Reynolds suggested that further modifications could lead to a herd model by giving the creatures the ability to follow spatial restriction.

The challenge is how to give the creatures the ability to follow spatial restriction while keeping the complexity low enough to still allow for real-time simulation of the herd. We would like the herd to move in natural-looking paths. Also, we would like the creatures to travel smoothly in 3D space, with speed regulation in curve.

Increasing use of autonomous unmanned air vehicles in a variety of civil and military applications is putting increasing pressure on traditional airspace management capabilities. One solution to this problem is decentralize the management function by delegating control such that individual aircraft manage their own airspace by negotiating with neighboring aircraft, i.e. free-flight. For 
example, two aircraft on a potential collision course will negotiate changes to their respective flight plans to remove the risk of collision. This negotiation takes place independently of a ground air traffic controller. However, for very high airspace densities, real time negotiation of alternative flight paths becomes impractical and it becomes necessary to impose a set of flight rules that minimizes the probability of collisions a priori.

In nature, aggregations of large numbers of mobile organisms are also faced with the problem of organizing themselves efficiently. This selective pressure has led to the evolution of behavior such as flocking of birds, swarming of insects, herding of land animals and schooling of fish. The reasons why organisms form flocks are varied and include protection from predation, improved food search and improved social cohesion. However, the actual dynamics of the flocking behavior are essentially constrained by the dynamics of the individual organisms and the flock is relatively limited in the types of behavior it can exhibit. This gives a flock of a given organism, be it fish or bird, its characteristic look and feel.

\section{Modeling of Flocking Behavior}

A flock may be loosely defined as a clustered group of individuals with a common velocity vector. Note that flocking of aircraft is different from formation flying. In the latter, aircraft are arranged according to predefined relationships that generally remain fixed during the flight. With flocking flight, there are no predefined relationships and the flock members may constantly change their position within the group. The fixed relationships within aircraft formations makes them relatively difficult to maneuver, whereas the fluid nature of a flock allows relatively rapid changes in flock direction.

The aim of the present work is to demonstrate flocking behavior of a group of simulated unmanned air vehicles. Of particular interest is the development of meaningful statistical metrics that usefully quantify flocking behavior and the investigation of the relationship between rule weighting and flocking behavior.

Underlying this type of motion control (steering objects around obstacles toward goals) is the concept of self-directed action. Rather than the traditional view of inert geometrical objects being moved according to a centralized, pre-existing plan (the script specified by the animator), here we consider the objects to be active independent entities capable of directing their own motion. They are not merely inert geometrical models but rather "self-motivated" behavioral models, which also happen to have a geometric component. Actually, most convenient are hybrid objects that can be directed in general terms from an animator's script but that can handle details of their own behavior as required. Specifically, the animator can set up goals for the behavioral objects, but the objects themselves will work out the specifics, such as the exact path that they take to avoid collisions with obstacles. Furthermore, the "guiding hand" of the animator is not required; behavioral objects 
are perfectly happy to merely wander around on their own, ad libbing their roles as "extras."

Formation behaviors in nature, like flocking and schooling, benefit the animals that use them in various ways. Each animal in a herd, for instance, benefits by minimizing its encounters with predators [3]. By grouping, animals also combine their sensors to maximize the chance of detecting predators or to more efficiently forage for food. Studies of flocking and schooling show that these behaviors emerge as a combination of a desire to stay in the group and yet simultaneously keep a separation distance from other members of the group [4]. Since groups of artificial agents could similarly benefit from formation tactics, robotics researchers and those in the artificial life community have drawn from these biological studies to develop formation behaviors for both simulated agents and robots. Approaches to formation generation in robots may be distinguished by their sensing requirements, their method of behavioral integration, and their commitment to preplanning. A brief review of a few of these efforts follows.

The viability of obtaining coherent flocking behavior from simple rules was first demonstrated by Reynolds [1]. The primary application for this work was in developing realistic motions of groups of 'actors' in computer animation. Conventionally, each 'actor' (known generically as a 'boid' in flocking work) has a scripted path predetermined by the animator. For large numbers of boids, for example a flock of birds, the process was cumbersome and did not produce realistic results. This led to the use of relatively simple flocking rules that would automatically govern the dynamic behavior.

Improvements to this approach have recently been made by $\mathrm{Tu}$ and Terzopoulos and separately by Brogan and Hodgins. Tu and Terzopoulos [5] developed more realistic simulated fish schooling by accurately modeling the animals' muscle and behavioral systems. Brogan and Hodgins [6] developed a system for realistically animating herds of one-legged agents using dynamical models of robot motion. Both results are more visually realistic than Reynolds' because they simulate the mechanics of motion; Reynolds' approach utilized particle models only.

The individual components of Reynolds' flocking and Bro-gan's herding behaviors are similar in philosophy to the motor schema paradigm used here, but their approaches are concerned with the generation of visually realistic flocks and herds for large numbers of simulated animals, a different problem domain than the one this article addresses. In contrast, our research studies behaviors for a small group (up to four) of mobile robots, striving to maintain a specific geometric formation.

The dynamics and stability of multi-robot formations have drawn recent attention [7], [8]. Wang [7] developed a strategy for robot formations where individual robots are given specific positions to maintain relative to a leader or neighbor. Sensory requirements for these robots are reduced since they only need to know about a few other robots. Wang's analysis centered on feedback control for formation maintenance and stability of the resulting system. It did not include integrative strategies for obstacle avoidance and navigation. In work by Chen and 
Luh [8] formation generation by distributed control is demonstrated. Large groups of robots are shown to cooperatively move in various geometric formations. Chen's research also centered on the analysis of group dynamics and stability, and does not provide for obstacle avoidance. In the approach forwarded in this article, geometric formations are specified in a similar manner, but formation behaviors are fully integrated with obstacle avoidance and other navigation behaviors.

Mataric has also investigated emergent group behavior [9], [10]. Her work shows that simple behaviors like avoidance, aggregation and dispersion can be combined to create an emergent flocking behavior in groups of wheeled robots. Her research is in the vein of Reynolds' work in that a specific agent's geometric position is not designated. The behaviors described in this article differ in that positions for each individual robot relative to the group are specified and maintained.

Related papers on formation control for robot teams include [11], [12], [13], [14]. Parker's thesis [11] concerns the coordination of multiple heterogeneous robots. Of particular interest is her work in implementing "bounding over watch," a military movement technique for teams of agents; one group moves (bounds) a short distance, while the other group over watches for danger. Yoshida [12], and separately, Yamaguchi [13], investigate how robots can use only local communication to generate a global grouping behavior. Similarly, Gage [14] examines how robots can use local sensing to achieve group objectives like coverage and formation maintenance. Parker simulates robots in a line-abreast formation navigating past waypoints to a final destination [15]. The agents are programmed using the layered subsumption architecture [5]. Parker evaluates the benefits of varying degrees of global knowledge in terms of cumulative position error and time to complete the task. Tucker and Ronald [17] presented reactive behavior for formation keeping and demonstrated successfully outdoors on cars.

\section{Boid for Simulating Flocking Behavior}

A method is presented for flocking behavior of creatures, birds, fishes and so on, that can form herds by evading obstacles in airspace, terrain or ocean floor topography in 3D space while being efficient enough to run in real-time. This method involves making modifications to Reynolds' flocking algorithm [2] as following.

- Cohesion: steer to move toward the average position of local flockmates

- Separation: steer to avoid crowding local flockmates

- Alignment: steer towards the average heading of local flockmates 
The boid is individual and it's behavior determines how a boid reacts to other characters in its local neighborhood, as shown grey area in Fig.1, 2 and 3. A boid outside of the local neighborhood are ignored.

Cohesion behavior gives a boid (outlined triangle located in the centre of the diagram) the ability to cohere with (approach and form a group with) other nearby boids. See Fig.1. Steering for cohesion can be computed by finding all characters in the local neighborhood (as described above for separation), computing the average position (or center of gravity) of the nearby characters. The steering force can applied in the direction of that average position (subtracting our character position from the average position, as in the original boids model). The flocking rules used in the present work are illustrated schematically. The cohesion rule, acts such that the active flock member (outlined triangle located in the centre of the diagram) tries to orient its velocity vector in the direction of the centroid (average spatial position) of the local flock. The degree of locality of the rule is determined by the sensor range of the active flock member, represented diagrammatically

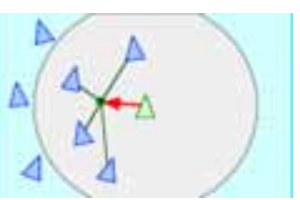

Fig.1 Cohesion

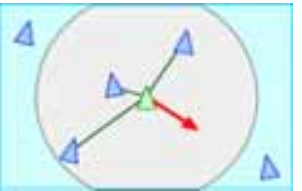

Fig.2 Separation

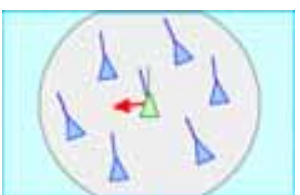

Fig.3 Alignment by the light colored circle. Note that for effective cohesion it is necessary to vary the speed of the active flock member as well as its heading such that the speed of boids far from the flock centroid is increased. This is referred to as speed cohesion and allows wayward boids to catch up with the rest of the flock.

Separation behavior gives a boid the ability to maintain a certain separation distance from others nearby. This can be used to prevent boids from crowding together. To compute steering for separation, first a search is made to find other characters within the specified neighborhood. This might be an exhaustive search of all characters in the simulated world, or might use some sort of spatial partitioning or caching scheme to limit the search to local characters. For each nearby character, a repulsive force is computed by subtracting the positions of our character and the nearby character, normalizing, and then applying a $1 / \mathrm{r}$ weighting. (That is, the position offset vector is scaled by $1 / r^{2}$.) Note that $1 / r$ is just a setting that has worked well, not a fundamental value. These repulsive forces for each nearby character are summed together to produce the overall steering force. See Fig.2.

Alignment behavior gives an character the ability to align itself with (that is, head in the same direction and/or speed as) other nearby characters, as shown in Fig.3. Steering for alignment can be computed by finding all characters in the local neighborhood (as described above for separation), averaging together the velocity (or alternately, the unit forward vector) of the nearby characters. This 
average is the desired velocity, and so the steering vector is the difference between the average and our boid's current velocity (or alternately, its unit forward vector). This behavior will tend to turn our character so it is aligned with its neighbors.

With simulations, we show that reasonable flocking behavior can be obtained using just cohesion and alignment rules.

Left unchecked, the cohesion rules will tend to lead to flock overcrowding. To balance this, a separation rule is used, where the active flock member tries to translate away from the local flock centroid. Note that for effective flocking behavior, the sensor range of the cohesion rules will generally be much larger than the separation rule, i.e. cohesion acts at a global level whereas separation works locally.

Each boid has direct access to the whole scene's geometric description, but flocking requires that it reacts only to flockmates within a certain small neighborhood around itself. The neighborhood is characterized by a distance (measured from the center of the boid) and an angle, measured from the boid's direction of flight. Flockmates outside this local neighborhood are ignored. The neighborhood could be considered a model of limited perception (as by fish in murky water) but it is probably more correct to think of it as defining the region in which flockmates influence a boids steering.

The modifications use only local properties of the spatial restriction, and thus have low complexity and wide application. The flocking algorithm with these modifications produces naturally behaving herds that follow the spatial restriction. The evading obstacles rule added to the flocking algorithm has a constant parameter that can be adjusted to produce different behaviors.

The flocking algorithm works as follows: For a given boid, centroids are calculated using the sensor characteristics associated with each flocking rule. Next, the velocity vector the given boid should follow to enact the rule is calculated for each of the rules. These velocity vectors are then weighted according to the rule strength and summed to give an overall velocity vector demand. Finally, this velocity vector demand is resolved in to a heading angle, pitch attitude and speed demand, which is passed to the control system. The control system then outputs an actuator vector that alters the motion of the aircraft in the appropriate manner.

\section{Simulation Results}

3D simulation have to be for flying behavior. We develop 3D simulation using Java 3D. Java 3D is an addition to Java for displaying three-dimensional graphics. 
Sometimes, Unmanned Vehicle fly through spatial restriction. There will be the Unmanned Vehicle's missions of flying low in the mountain area. We study that Unmanned Vehicles fly through spatial restriction with flocking. Some case, fly through narrow canyon (Fig.4). Fig.5 is a simulation result of the case. Some other case, fly through some walls (Fig.6).

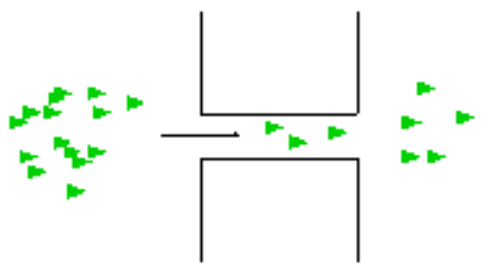

Fig.4 Flying Through Narrow Canyon

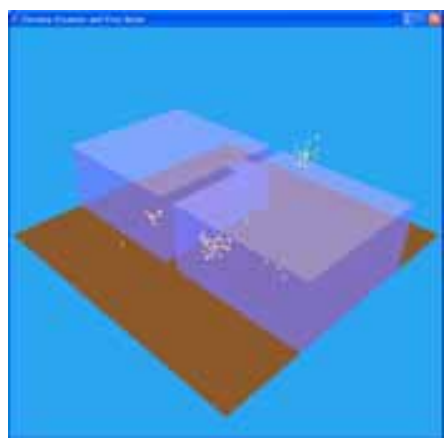

Fig.5 The Snap of Simulation Result of Flying through Narrow Canyon

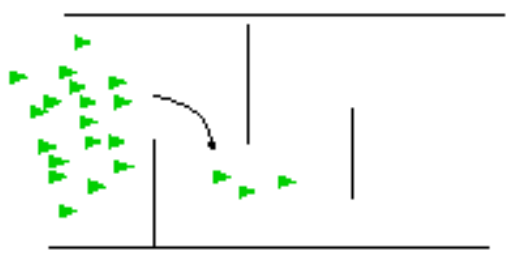

Fig.6 Flying through Walls

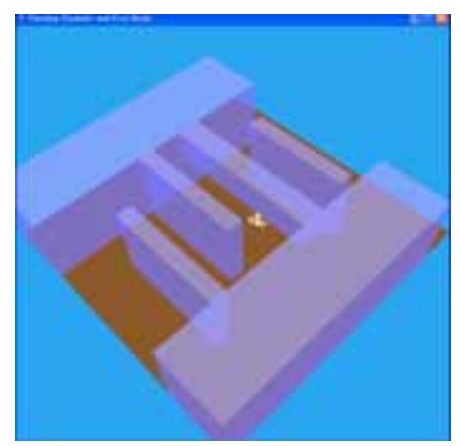

Fig.7 The Snap of Simulation Result of Flying Through Walls

Simulation results will be presented to show the effect of changing rule weightings. The baseline test case is a flock initially placed at random positions and orientations. Heading, attitude and velocity guidance demand vectors for each boid based on flocking rules are calculated. Note simulation time increases linearly with increasing guidance update rate and exponentially with number of boids.

\section{Design of Collective Intelligence}

Today we have many challenges for designing large-scale and complex systems consisting of multiple physically and geographically distributed processors. Such systems offer the promise of speed, reliability, extensibility and the potential for increased tolerance to uncertain date and knowledge. The conventional approach requires a unifying control or coordination mechanism in order to extend the 
partial views and the incomplete knowledge of components and to guide a global solution. By true meaning of distribution that both control and knowledge are physically and often geographically distributed, we mean that there is neither global control nor global knowledge storage. There are two methodologies that need to be developed to address this view of complex systems. First, distributed coordination mechanisms that enable systems to organize themselves and accomplish critical tasks with available resources. Second, self-organizing techniques that enable systems to improve with experience themselves.

Collective means any pair of a complex system of autonomous components, together with a performance criterion by which we rank the behavior of the overall system. Any given collective phenomena can be viewed as an emergent, largescale, long-term entity that has arisen from billions of small-scale, short-term actions of individual agents, that is, as a complex adaptive system. The performance of the collective system consisting of interacting agents depends on how agents are properly coupled. In examining collective, we shall draw heavily on the individual adaptive behavior. To make that connection we usually have to look at how interacting agents adapt to others.

The design of efficient collectives from bottom up becomes is an important issue in many areas. Collective means any pair of a complex system of autonomous components, together with a performance criterion by which we rank the behavior of the overall system. The performance of the collective system which consists of many interacting agents should be described on two different levels: the microscopic level, where the decisions of the individual agents occur and the macroscopic level where collective behavior can be observed. To understand the role of a link between these two levels remains one of the challenges of complex system theory.

Wolpert and Tumer [19] propose that the fundamental issue is to focus on improving our formal understanding of two closely related issues concerning collective:

(1) The forward problem of how the fine-grained structure of the system underlying a collective determines its complex emergent behavior and therefore its performance.

(2) The inverse problem of how to design the structure of the system underlying a collective to induce optimal performance.

Agent-based model involves the study of many independent agents and their interactions. It is regarded as a new way of doing science through experiments. Like deduction, it starts with a set of explicit assumptions. But unlike deduction, it does not prove theorems. Instead, an agent-based model generates simulated data that can be analyzed inductively. Unlike typical induction, however, the simulated data come from a rigorously specified set of rules rather than direct measurement of the real world. When the agents use adaptive rather than optimizing strategies, 
deducing the consequences is often impossible, and then simulation becomes necessary. We specify how the agents interact, and then observe properties that occur at the macro level. The connection between micro-motivation and macrooutcomes will be developed through agent-based simulation, in which a population of agents is instantiated to interact according to fixed or evolving rules of behavior. The question of whether interacting agents self-organize efficient macroscopic orders from bottom up depends on how they interact each other. We attempt to probe deeper understanding this issue by specifying how they interact each other.

The two most important attributes of a flock were found to be its density (how close its members are to each other) and the degree of alignment of member velocity vectors. For the present work flock density is represented by the mean radius between flock members, and flock alignment by the standard deviation of the flock member heading angles.

\section{Conclusion}

Flocking offers a potentially simple and efficient way of managing the flight paths of a large number of small autonomous UAVs such that the risk of collision and/or the need for evasive maneuvers is reduced. The way in which flocking rules are implemented depends strongly on the nature of the flight control system available on the target flight vehicle. We will evolve flying through spatial restriction. We want to manage boid flock to fly through maze in future.(Fig. 8)

\section{References}

[1] Reynolds, C. W. (1987) "Flocks, Herds, and Schools: A Distributed Behavioral Model, in Computer Graphics," 21(4) (SIGGRAPH '87 Conference Proceedings) , pp.25/34.

[2] Reynolds, C. W. (1999) "Steering Behaviors For Autonomous Characters," in the proceedings of Game Developers Conference 1999 held in San Jose, California. Miller Freeman Game Group, San Francisco, California. pp.763/782.

[3] Hertz J., Krogh A., Palmer, R. G. (1991) "Introduction to the Theory of Neural Computation," Addison -Wesley.

[4] S. L. Veherencamp (1987) "Individual, kin, and group selection," in Handbook of Behavioral Neurobiology, Volume 3: Social Behavior and Communication, P. Marler and J. G. Vandenbergh, Eds. New York: Plenum, pp. 354/382. 
[5] J. M. Cullen, E. Shaw, and H. A. Baldwin (1965) "Methods for measuring the three-dimensional structure of fish schools," Animal Beh., vol.13, pp.534/543

[6] X. Tu and D. Terzopoulos (July 1994) "Artificial fishes: Physics, locomotion, perception, behavior," in Proc. SIGGRAPH 94 Conf., Orlando, FL, pp.43/50

[7] D. C. Brogan and J. K. Hodgins (Mar. 1997) "Group behaviors for systems with significant dynamics," Auton. Robots, vol. 4, no. 1, pp.137/153

[8] P. K. C. Wang (1991) "Navigation strategies for multiple autonomous robots moving in formation," J. Robot. Syst., vol. 8, no. 2, pp.177/195

[9] Q. Chen and J. Y. S. Luh (1994) "Coordination and control of a group of small mobile robots," in Proc. IEEE Int. Conf. Robot. Automat., San Diego, CA, 1994, pp. 2315/2320

[10] M. Mataric (1992) "Designing emergent behaviors: From local interactions to collective intelligence," in Proc. Int. Conf. Simulation of Adaptive Behavior: From Animals to Animats 2, pp.432/441

[11] (May 1992) "Minimizing complexity in controlling a mobile robot population," in Proc. 1992 IEEE Int. Conf. Robot. Automat., Nice, France, pp. 830/835

[12] L. E. Parker (1994) "Heterogeneous Multi-Robot Cooperation," Ph.D. dissertation, Dept. Electr. Eng. Comput. Sci., Mass. Inst. of Technol., Cambridge, MA

[13] E. Yoshida, T. Arai, J. Ota, and T. Miki (1994) "Effect of grouping in local communication system of multiple mobile robots," in Proc. 1994 IEEE Int. Conf. Intell. Robots Syst., Munich, Germany, pp. 808/815

[14] H. Yamaguchi (Apr. 1997) "Adaptive formation control for distributed autonomous mobile robot groups," in Proc. 1997 IEEE Conf. Robot. Automat., Albuquerque, NM

[15] D. W. Gage (1992) "Command control for many-robot systems," Unmanned Syst. Mag., vol. 10, no. 4, pp. 28/34

[16] L. Parker (1993) "Designing control laws for cooperative agent teams," in Proc. 1993 IEEE Int. Conf. Robot. Automat., pp. 582/587

[17] R. Brooks (Feb. 1986) "A robust layered control system for a mobile robot," IEEE J. Robot. Automat., vol. RA-2, p. 14

[18] Tucker Balch and Ronald C. Arkin (Dec 1998) "Behavior-Based Formation Control for Multirobot Teams," IEEE Transactions on Robotics and Automation, vol.14, no.6

[19] Wolpert and Tumer (2004) "Collectives and the Design of Complex Systems," Springer. 